\title{
Comparación de máscaras laríngeas (I-gel vs Fastrach) para realización de intubación endotraqueal guiada con broncofibroscopio flexible
}

\section{Comparison of I-gel vs Fastrach laryngeal masks in conducting flexible bronchofiberscope-guided}

\author{
Jonatán A. Díaz MD. ${ }^{1}$, Germán Cappa MD. ${ }^{2}$, Franco A. Pugnaloni MD. ${ }^{2}$, Guillermina B. Harvey³
}

\begin{abstract}
Goals: Determine the success rate of guided intubation with a flexible bronchofibroscope (BFC) through supraglottic devices: I-gel or Fastrach, establish the degree of fibroscopic visualization and determine the insertion time of the laryngeal mask airway (LMA) and endotracheal intubation. Methods: Experimental, prospective, comparative and randomized study of 60 ASA I-II-III patients, older than 18 years, undergoing elective surgeries that required general anesthesia and endotracheal intubation. After anesthetic induction, the assigned LMA was placed and intubation with BFC was performed through it. Both the success of laryngeal mask insertion and intubation were recorded, as well as the time (in seconds) equired for these procedures. The vision of the laryngeal structures was classified according to the Brimacombe Scale. Results: The groups analyzed presented similar general characteristics. Insertion time of he LMA was significantly lower with I-gel than Fastrach (median $12 \mathrm{~s}$ vs 16 s. Respectively, $p=0.001$ ). Also, in the intubation time BFC guided through the laryngeal mask, differences in favor of I-gel were found, with a median of 72 s. vs 89 s. $(p=0.001)$. Regarding the degree of glottic visualization with BFC, no statistically significant differences were found between both groups.
\end{abstract}

\section{Key words:}

Intubation,

intratracheal,

laryngeal masks, airway managemen, anesthesia general

Médico Anestesiólogo. Hospital Provincial de Rosario. Universidad Nacional de Rosario.

Médico Anestesiólogo. Hospital Provincial de Rosario. Universidad Nacional de Rosario. Instructor de Simulación Clínica.

Magíster en Estadística Aplicada. Carrera de Anestesiología. Facultad de Ciencias Médicas. Universidad Nacional de Rosario.

Fecha de recepción: 01 de octubre de 2020

Fecha de aceptación: 10 de enero de 2021

\section{ORCID}

0000-0001-8716-6801

Fuente de financiamiento: El trabajo fue realizado sin financiamiento externo.

Conflicto de intereses: Los autores de este estudio, declaran no tener conflicto de intereses.

\section{Correspondencia:}

Franco A. Pugnaloni

Pugnaloni.franco@gmail.com 
Conclusions: Although both devices had the highest success rate (both in their placement and in intubation through them), with I-gel the intubation was performed quicker.

\section{RESUMEN}

Objetivos: Determinar la tasa de éxito de intubación guiada con broncofibroscopio flexible (BFC) a través de los dispositivos supraglóticos: I-gel o Fastrach, establecer el grado de visualización fibroscópica y determinar el tiempo de inserción de la máscara laríngea y de intubación endotraqueal. Materiales y Métodos: Estudio experimental, prospectivo, comparativo y aleatorizado de 60 pacientes ASA I- II -III, mayores de 18 años, sometidos a cirugías electivas que requerían anestesia general e intubación endotraqueal. Luego de la inducción anestésica, se procedió a colocar la máscara laríngea asignada y se realizó la intubación con BFC a través de la misma. Tanto el éxito de inserción de máscara laríngea como de intubación fueron registrados, así como el tiempo (en segundos) requerido para dichos procedimientos. La visión de las estructuras laríngeas fue clasificada de acuerdo a Escala de Brimacombe. Resultados: Los grupos analizados presentaron características generales similares. El tiempo de inserción de la ML fue significativamente menor con I-gel que con Fastrach (medianas: $12 \mathrm{~s}$. vs $16 \mathrm{~s}$. respectivamente, $\mathrm{p}=0,001$ ). También en el tiempo de intubación guiada con BFC a través de la máscara laríngea, se encontraron diferencias en favor de I-gel, con una mediana de 72 s. vs 89 s. $(p=0,001)$. Respecto al grado de visualización glótica con BFC, no se encontraron diferencias estadísticamente significativas entre ambos grupos. Conclusiones: Aunque ambos dispositivos tuvieron la máxima tasa de éxito (tanto en su colocación como en la intubación a través de ellos), con I-gel se realizó la intubación de manera más rápida.

\section{Palabras clave:}

Intubación intratraqueal, máscaras laríngeas, manejo de la vía aérea, anestesia general

\section{Introduccón}

I manejo de la vía aérea constituye una de las bases fundamentales de la asistencia del paciente crítico y de la práctica anestesiológica. Una de las situaciones con las cuales se encuentra el anestesiólogo cotidianamente, y a la cual se le imparte un gran respeto, es la de la intubación dificultosa.

La ASA (American Society of Anesthesiologists) define intubación dificultosa como aquella situación en la que un anestesiólogo con experiencia, usando laringoscopia directa requiere: 1) más de 2 intentos con una misma hoja de laringoscopio; 2) un cambio de hoja o una ayuda a la laringoscopia directa (por ejemplo: guía de Eschmann, fiador, etc.); 3) uso de un sistema o técnica alternativa tras un fallo en la intubación con laringoscopia directa[1],[2].

La vía aérea dificultosa (VAD) no anticipada ocurre con una baja incidencia en la práctica anestesiológica; sin embargo, su presencia conlleva a eventos verda- deramente catastróficos. La dificultad en la realización de la laringoscopía directa, ocurre en 1,5\%-8\% de las anestesias generales, manteniendo una similar incidencia la intubación dificultosa. Cabe destacar, que la falla en la intubación ocurre en el 0,13\%-0,3\% de las mismas[3].

Algunas de las principales causas de VAD son laringoscopía difícil, obesidad, limitación de la flexoextensión de la columna cervical, artritis reumatoidea, macroglosia, micrognatia, apertura bucal limitada, tumores en región oral y nasofaríngea, síndrome de apnea obstructiva del sueño, compresión o estenosis de la vía aérea, entre otras; sin embargo, gracias al examen físico y diversos tests es posible, en muchas ocasiones, divisarla y anticipar su abordaje[4],[5].

Desde la introducción en el mercado en 1988, gracias a su mentor Archie Brain, la máscara laríngea (ML) es considerada una alternativa en el manejo de la $V A D$, principalmente en lo que respecta a la ventilación dificultosa. Desde entonces, los dispositivos 
supraglóticos han cobrado una gran importancia, y han sido actualizados e innovados para adquirir nuevas propiedades. De los existentes en el mercado, se utilizarán dos: en un grupo se empleará ML I-gel y en el otro Fastrach[6],[7)].

La ML l-gel es un dispositivo creado por el Dr. Muhammed Aslam Nasir, fabricado de un elastómero termoplástico diseñado para adaptarse a las estructuras perilaríngeas e hipofaríngeas, sin necesidad de utilizar un manguito inflable.

Es una ML considerada de segunda generación, que se adapta armónicamente a la anatomía del paciente, de forma que se reducen significativamente las compresiones y desplazamientos traumáticos[8]. Se utiliza para estabilizar y mantener la vía respiratoria del paciente durante los procedimientos anestésicos de rutina y de urgencias en aquellas intervenciones que requieren ventilación espontánea o ventilación por presión positiva intermitente. Incorpora un canal gástrico que permite la succión, el paso de sondas gástricas y facilita la ventilación. Reduce la posibilidad de oclusión de la vía aérea, estabiliza la cavidad bucal, proporciona la inserción, y elimina el riesgo de rotación.

I-gel se suministra en un envase de polipropileno con código de colores para una rápida y fácil identificación. Está disponible en siete tamaños: cuatro para pediatría y tres para adultos[9].

La ML de intubación Fastrach, fue por primera vez desarrollada por el Dr. A. Brain en 1997, como solución a la dificultad encontrada al intentar insertar un tubo endotraqueal (TET) a ciegas, a través de la mascarilla laríngea clásica. Está diseñada para permitir la ventilación, así como también la intubación.

Las principales características que diferencian a la ML Fastrach de la ML Clásica son:

1. Presencia de un tubo rígido anatómicamente precurvado.

2. Presencia de mango metálico.

3. Barra elevadora de la epiglotis (BEE) (que sustituye a las barras de apertura vertical de la ML Clásica).

4. Rampa-guía construida en el suelo de la cazoleta de la ML.

El conjunto de estas características, permiten el óptimo alineamiento de esta ML con la abertura glótica para el paso del TET. Se encuentra disponible en 3 tamaños, utilizados de acuerdo al peso del paciente.

Puede ser utilizada combinada con otras técnicas como la fibrobroncoscopía y las técnicas de transiluminación en pacientes con criterios de dificultad en el control de la vía aérea con tasas de éxito elevadas (entre el $99 \%$ y el $100 \%$ para la primera combinación y el $95 \%$ y el $100 \%$ para la segunda). La inserción de la
ML Fastrach posee un elevado índice de éxito (95\%$100 \%)$, al igual que en la intubación orotraqueal "a ciegas" (81\%-100\%)[10].

El broncofibroscopio (BFC) sigue siendo considerado de referencia actualmente para el manejo de la VAD. Es un dispositivo muy valorado, y en general se considera a la intubación fibroóptica en estado vigil como la primera elección en casos de vía aérea difícil conocida, requiriendo un acondicionamiento previo del paciente y preparación del especialista a cargo. Su utilización requiere una formación adecuada siendo ésta, además de la disponibilidad de equipos y la experiencia de los operadores, sus principales limitantes en nuestro medio. Sin embargo, es una técnica cada vez más utilizada, por lo que se recomienda que todos los anestesiólogos estén familiarizados con su utilización. El sangrado en la vía aérea y las secreciones dificultan su implementación, limitando su uso en urgencias con gran apremio de tiempo, siendo este último la variable fundamental en estas circunstancias.

Debido a esto, surge la necesidad de comenzar a hablar de "combinación de dispositivos", tal como lo demuestran los trabajos Sastre y col.[11], de Lloyd y col.[12] y Michalek y col.[13] para, de esta manera, aumentar las chances de éxito que cada uno ofrece por separado: intubación a ciegas a través de la ML o intubación con BFC flexible sin ningún dispositivo guía.

Podría pensarse en la posibilidad de intubación en pacientes vigiles en aquellos en quienes se sospecha una vía aérea dificultosa, pero su práctica es muy compleja y necesita una sólida y continua formación.

De esta forma se pretende brindar herramientas alternativas a aquellas personas que dispongan de BFC flexible, pero cuya curva de aprendizaje no sea la adecuada. Se da la oportunidad de demostrar que es posible la intubación endotraqueal a través de una $\mathrm{ML}$ (sea o no considerada intubadora), guiada con BFC, para así aumentar las chances de éxito[14]. Se deja también entrever que si, ambas ML permiten una ventilación adecuada, también deberían ser capaces de guiarnos hacia la glotis.

Teniendo en cuenta lo expuesto anteriormente, la hipótesis que incentivó este trabajo fue que la l-gel (máscara laríngea considerada no intubadora) sería mejor que la Fastrach (máscara laríngea intubadora) para realizar intubación endotraqueal guiada a través de BFC.

\section{Objetivo general}

- Determinar la tasa de éxito de intubación guiada con broncofibroscopio flexible a través de los dispositivos: I-gel o Fastrach. 


\section{Objetivos secundarios}

- Evaluar el porcentaje de éxito en el primer intento.

- Determinar el tiempo de inserción de la máscara laríngea.

- Establecer el grado de visualización fibroscópica (escala de Brimacombe).

- Determinar el tiempo de intubación.

\section{Materiales y Métodos}

Con la aprobación del Comité de Bioética del Hospital Provincial de Rosario, provincia de Santa Fe, Argentina, se llevó a cabo en ese hospital un estudio experimental, prospectivo, comparativo y aleatorizado durante el período comprendido entre los meses de agosto de 2016 y enero de 2017, en el marco de la tesis final de uno de los autores para finalizar su formación en Anestesia, Analgesia y Reanimación, en el posgrado realizado en la Asociación Rosarina de Anestesiología.

Se obtuvo la autorización a través del consentimiento informado de 60 pacientes ASA I-II-III, mayores de 18 años, sometidos a cirugías electivas que requerían anestesia general e intubación endotraqueal. Los pacientes fueron asignados a uno de dos grupos siguiendo una lista de aleatorización generada por un investigador externo. Los grupos se determinaron de acuerdo a la máscara laríngea utilizada: I-gel (grupo I-Gel) o Fastrach (grupo Fastrach).

Se excluyeron los pacientes con una apertura bucal menor a $2 \mathrm{~cm}$, presencia de factores de riesgo de aspiración gástrica (hernia de hiato, historia de cirugía gástrica o esofágica), contraindicación del uso de relajantes neuromusculares, y alergia conocida a alguna de las drogas utilizadas.

Una vez que el paciente se encontraba en quirófano, se realizó una monitorización estándar (según la Sociedad Americana de Anestesiología) y correcto posicionamiento: decúbito dorsal, a $180^{\circ}$ en la camilla, con cabeza apoyada sobre una almohada para mantener una posición cervical neutral y alineación de ejes buco-faringo-laríngeo. Se procedió a desnitrogenación durante 1 minuto con oxígeno 100\% con circuito lineal (Mapleson C).

Seguidamente se dio comienzo a la inducción anestésica utilizando:

- Propofol: 1,5-2,5 mg/kg en bolo.

- Fentanilo: $2-3 \mu \mathrm{g} / \mathrm{kg}$.

- Remifentanilo: 0,2-0,3 $\mu \mathrm{g} / \mathrm{kg} / \mathrm{min}$.

Previo a la realización del relajante neuromuscular (vecuronio 0,8 $\mathrm{mg} / \mathrm{kg}$ ), se comprobó que la ventilación con máscara facial fuese la adecuada. Los pacientes se continuaron ventilando por un minuto para dar tiempo a la acción de dicho fármaco.

Se insertó la ML correspondiente de acuerdo al peso del paciente (Tablas 1 y 2 ). Se siguieron las pautas propuestas por su fabricante para su colocación[19],[20]. Se comprobó su correcto posicionamiento mediante tres pruebas: expansión torácica, presencia de curva normal de capnografía y ausencia de sonido audible que indique fuga insuflando a una presión que oscile entre 10 y $20 \mathrm{cmH}_{2} \mathrm{O}$ durante ventilación manual. Se cronometró el tiempo requerido para su correcta inserción (tiempo 1).

En los casos en los que la ventilación fue ineficaz,

\begin{tabular}{lccc}
\multicolumn{5}{c}{ Tabla 1. Tamaño de ML Fastrach, volumen máximo de inflado de su manguito y tamaño del TET recomendados } \\
de acuerdo al peso del paciente
\end{tabular}

\begin{tabular}{|c|c|c|}
\hline Peso del paciente $(\mathrm{kg})$ & Tamaño de ML I-gel recomendado & Tamaño del TET recomendado \\
\hline $30-50$ & 3 & 7 \\
\hline $50-90$ & 4 & 7,5 \\
\hline$>90$ & 5 & 8 \\
\hline
\end{tabular}


se corrigió el posicionamiento de la ML; en el caso en que esto no hubiese funcionado, se hubiese elegido una de menor o mayor tamaño en base a consideraciones anatómicas y al criterio del anestesiólogo a cargo.

Luego de comprobar la correcta ventilación a través del dispositivo supraglótico, se eligió el TET descartable que mejor se adecuó a cada paciente de acuerdo al tamaño de ML utilizado. El mismo fue montado a un BFC flexible (Olympus BF type 1t30, diámetro exterior de 5,8 mm), previamente a ser lubricado con xilocaína en jalea, para permitir su correcto deslizamiento a través del canal de la ML.

Se introdujo el BFC, con el tubo lubricado y montado, a través de la ML. Al llegar al orificio de salida, se visualizaron las estructuras laríngeas y se puntuaron de acuerdo a la clasificación de Brimacombe[21]. Dicha clasificación determina por vía endoscópica el grado de visualización según la posición de la ML, asignando una puntuación de 4 a 0 de la siguiente manera:

4: Visualización exclusiva de las cuerdas vocales.

3: Visualización de las cuerdas vocales y la epiglotis posterior.

2: Visualización de las cuerdas vocales y la epiglotis anterior.

1: No se visualizan las cuerdas vocales pero la ventilación es adecuada.

0: Falla en la ventilación con ausencia de visión endoscópica de las cuerdas vocales.

Se pasó el BFC a través de las cuerdas vocales, hasta lograr visualizar la carina traqueal. En ese momento, con la cooperación de un ayudante, se progresó el TET hasta que se confirmó su correcta inserción en la tráquea. Se retiró el BFC y quedó confirmada la intubación con la aparición de una curva de capnografía normal. Se cronometró el tiempo de este proceso.

Seguidamente se procedió al retiro del dispositivo supraglótico, para lo cual se utilizó en ambos dispositivos la ayuda de un tubo estabilizador. Una vez retirado el mismo, se procedió a ventilar al paciente, verificando de esa manera el correcto posicionamiento del TET. Se cronometró este tiempo que, sumado al anterior, se denominó tiempo 2.

Se analizaron las siguientes variables basales: edad (años), sexo, IMC (peso/talla²), clasificación de Mallampatti, distancia tiro-mentoniana $(\mathrm{cm})$ y la apertura bucal $(\mathrm{cm})$. Dentro de las variables específicas se analizó: ML utilizada, éxito en el primer intento de colocación de ML, tiempo de inserción de ML (seg), tiempo de intubación con BFC (seg), grado de visualización glótica con BFC según Brimacombe, porcen- taje de saturación registrado en 3 momentos (luego de colocada la ML, luego de la inserción del TET y luego de retirada la $\mathrm{ML}$ ) y la medición de capnometría luego de retirada la ML (como variable de tiempo de duración)[15],[16].

Tres intentos fueron permitidos tanto para la inserción y la correcta posición de la ML como para la intubación endotraqueal a través de la misma. En caso de falla de los mismos, se procedería al manejo de la vía aérea con un método alternativo (a cargo del anestesiólogo presente). Además, durante la realización de cualquiera de los procedimientos, en caso de ser necesario, se pudo reanudar la ventilación a través de mascara facial o ML manualmente para mantener la saturación de oxígeno superior a $92 \%$ en todo momento. Se consideró desaturación cuando fue menor a $92 \%$. Se previó el registro, en caso de presentarse, de estos eventos, así como de cualquier otro (laringoespasmo, broncoespasmo, etc.). Cabe aclarar que el investigador del estudio tenía experiencia similar con ambos dispositivos supraglóticos, y fue el único operador involucrado tanto en la técnica de los procedimientos como en la medición de los resultados.

\section{Análisis estadístico}

Los datos se presentan como promedio (desvío es-

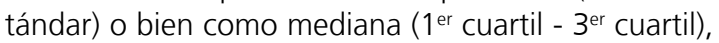
de acuerdo a la distribución de los datos, en el caso de las variables continuas. Para las variables categóricas, se indican las frecuencias $\left(n^{\circ}\right)$ junto con los porcentajes (\%).

En la comparación de las variables continuas se utilizó el test de la $U$ de Mann-Whitney al no cumplirse el supuesto de normalidad, lo cual fue evaluado mediante el test de Kolmogorov-Smirnov. En el caso de las variables nominales, para comparar las proporciones entre los grupos, se utilizó el test Chi-cuadrado de Pearson o bien el test de Fisher. En la evaluación de las variables ordinales se utilizó el test de los scores medios. Los resultados con una probabilidad asociada menor que 0,05 se consideraron estadísticamente significativos.

\section{Resultados}

Un total de 60 pacientes fueron ingresados y analizados en el estudio, 30 en cada grupo. No se encontraron diferencias estadísticamente significativas en las características generales entre ambos grupos (Tabla 3).

Todos los pacientes tuvieron un solo intento de 


\begin{tabular}{lccc}
\multicolumn{4}{c}{ Tabla 3. Características generales de la muestra } \\
\hline & Grupo I-GEL $(\mathbf{n}=\mathbf{3 0})$ & Grupo FASTRACH $(\mathbf{n}=\mathbf{3 0})$ & $\mathbf{p}$ \\
Edad (años) & $39,8(15,4)$ & $34,5(11,3)$ & 0,248 \\
Peso $(\mathrm{k})^{\mathrm{a}}$ & $75,1(13,1)$ & $75,4(15,2)$ & 0,894 \\
Talla $(\mathrm{m})^{\mathrm{a}}$ & $1,7(0,1)$ & $1,7(0,1)$ & 0,837 \\
IMC a & $26,8(3,6)$ & $27,2(4,8)$ & 0,976 \\
Sexo (femenino) & $18(60 \%)$ & $21(70 \%)$ & 0,417 \\
\hline
\end{tabular}

Los datos se presentan como: ${ }^{a}$ promedio (desvío estándar); probabilidad asociada al test U de Mann-Whitney; ${ }^{b} n^{\circ}(\%)$ - probabilidad asociada al test Chi-cuadrado de Pearson.

colocación de ML.

El tiempo de inserción de la ML fue significativamente menor con I-gel que con Fastrach $(p=0,001)$. Mientras en el grupo l-gel el $50 \%$ de la muestra se concentra entre 10 y $14 \mathrm{~s}$, en el grupo Fastrach, el $50 \%$ se agrupa entre 14 y $20 \mathrm{~s}$ (medianas: 12 s vs 16 $s$ respectivamente) (Figura 1).

En cuanto al tiempo de intubación guiada con BFC a través de la $\mathrm{ML}$, también se encontraron diferencias estadísticamente significativas en favor de I-gel, con un $50 \%$ de la muestra que se concentra entre los 63 y 79 s (rango: 47-142 s). Por el otro lado, en el grupo Fastrach, el $50 \%$ se agrupa entre 85 y 105 s (rango: 55-257 s) (mediana de 72 vs 89 s. respectivamente, $p$ $=0,001$ ) (Figura 2).

No se presentaron episodios de desaturación durante los momentos de colocación y retirada de las ML; no obstante, sí se registró un episodio de desaturación asociado a broncoespasmo luego de la colocación del TET a través de Fastrach, que revirtió con ventilación manual.

Las diferencias en ambos grupos en relación a la medición de la capnometría luego de retirada la $\mathrm{ML}$ no fueron estadísticamente significativas $(p=0,386)$ (Tabla 4).

\section{Discusión}

Los resultados de este estudio demostraron que tanto las ML Fastrach como I-gel son útiles y seguras para realizar intubación endotraqueal guiada con $\mathrm{BFC}$, presentando esta última menores tiempos tanto en su colocación como en la intubación a través de ella. Si bien las características anatómicas que permiten predecir una vía aérea dificultosa fueron similares en ambos grupos, estudios demuestran que las mismas son de suma importancia tanto a la hora de la colocación de una ML como de la intubación a través de ellas. Por ejemplo, Tentillier et al.[17] evidencian la realización de múltiples intentos o el fallo en la colocación en pacientes con criterios clínicos de vía aérea dificultosa.

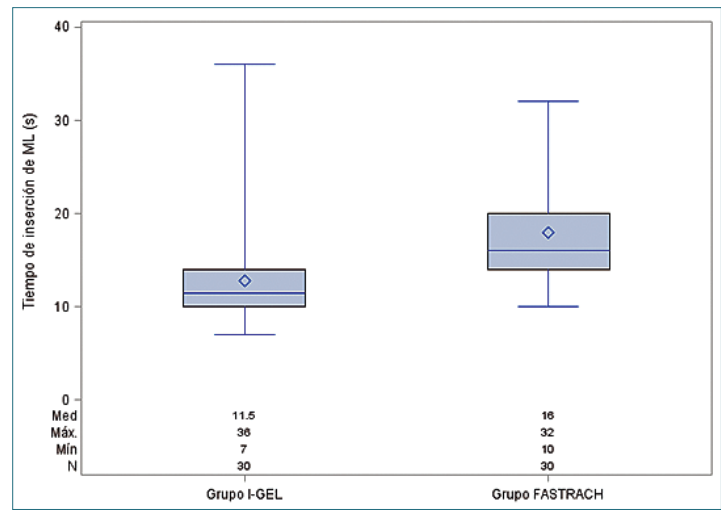

Figura 1. Distribución del tiempo de inserción de ML (s) según grupo.

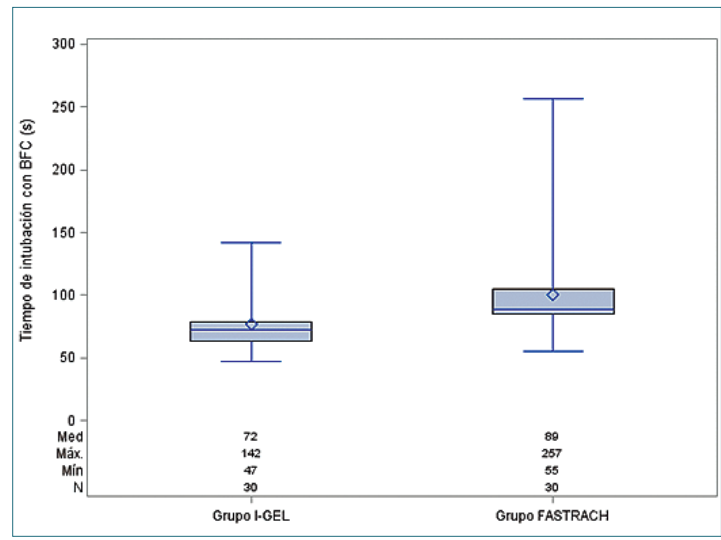

Figura 2. Distribución del tiempo de intubación con BFC (s) según grupo. 


\begin{tabular}{|c|c|c|c|}
\hline & $\begin{array}{l}\text { Grupo I-GEL } \\
\quad(n=30)\end{array}$ & $\begin{array}{l}\text { Grupo FASTRACH } \\
(\mathbf{n}=30)\end{array}$ & $\mathbf{p}$ \\
\hline Distancia tiromentoniana $(\mathrm{cm})^{\mathrm{a}}$ & $6(6-7)$ & $6(6-7)$ & $\begin{array}{l}0,635 \\
0,785\end{array}$ \\
\hline $\begin{array}{l}\text { Mallampattib } \\
1 \\
2 \\
3\end{array}$ & $\begin{array}{c}19(64 \%) \\
10(33 \%) \\
1(3 \%)\end{array}$ & $\begin{array}{c}17(57 \%) \\
11(37 \%) \\
2(6 \%)\end{array}$ & \\
\hline $\begin{array}{l}\text { Apertura bucal }(\mathrm{cm})^{b} \\
2 \\
3 \\
4\end{array}$ & $\begin{array}{c}0(0 \%) \\
24(80 \%) \\
6(20 \%)\end{array}$ & $\begin{array}{c}2(6 \%) \\
20(67 \%) \\
8(27 \%)\end{array}$ & 0,272 \\
\hline $\begin{array}{l}\text { Grado de visualización glótica con } \mathrm{BFC}^{\mathrm{b}} \\
1 \\
2 \\
3 \\
4\end{array}$ & $\begin{array}{l}1(3 \%) \\
2(6 \%) \\
5(17 \%) \\
22(74 \%)\end{array}$ & $\begin{array}{c}0(0 \%) \\
5(17 \%) \\
11(37 \%) \\
14(46 \%)\end{array}$ & 0,102 \\
\hline Tiempo de inserción de ML (s)a & $12(10-14)$ & $16(14-20)$ & 0,001 \\
\hline $\begin{array}{l}\text { Desaturación luego de la inserción del } \\
\text { TET }^{c}\end{array}$ & $0(0 \%)$ & $1(3 \%)$ & 0,999 \\
\hline Tiempo de intubación con BFC (s)a & $72(63-79)$ & $89(85-105)$ & 0,001 \\
\hline $\begin{array}{l}\text { Medición de capnometría luego de reti- } \\
\text { rada la } \mathrm{ML}^{\mathrm{a}}\end{array}$ & $27(25-30)$ & $25(22-29)$ & 0,386 \\
\hline
\end{tabular}

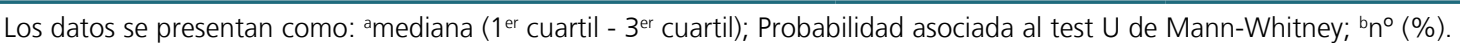
Probabilidad asociada al test de los scores medios; ${ }^{c} n^{\circ}(\%)$. Probabilidad asociada al test de Fisher.

El tiempo necesario para la colocación de las ML mostró diferencias estadísticamente significativas entre grupos, con una mediana de 12 segundos para I-gel, y de 16 segundos para Fastrach. Estos tiempos son menores a los publicados por Moore et al.[14], quienes registran un tiempo de 17 y 27 segundos, respectivamente. Se puede apreciar en ambos trabajos, el menor tiempo requerido para la colocación de una ML en poblaciones similares. Asimismo, el tiempo de intubación mostró diferencias estadísticamente significativas, con un tiempo mediano en el grupo Igel de 72 s vs 89 s en el grupo Fastrach. Estos tiempos son mayores a los observados por Moore et al.[14], ya que, en el presente trabajo, se registró el tiempo desde el momento que se ingresaba con el BFC a través del canal de la ML hasta la retirada del dispositivo supraglótico. Se analizó de este modo debido a que esta última maniobra podría producir algún evento desfavorable, como la extubación del paciente. Además, pueden presentarse cirugías en las cuales la ML debe ser retirada para que el cirujano trabaje con mayor comodidad.
El grado de visualización de estructuras glóticas fue superior con la ML I-gel, correlacionándose con el encontrado por Moore et al.[16], en concordancia tanto con Sastre et al.[11] como con Theiler et al.[18].

La tasa de intubación a través de ambas ML guiada con BFC fue del $100 \%$ en los pacientes analizados, mientras que en el estudio de Moore et al[14], la tasa de éxito con I-gel fue superior a la de Fastrach. De igual manera, en el trabajo publicado por Michalek et al.[13] en el cual se comparan el éxito de intubación a ciegas o guiada por BFC en maniquíes a través de dos ML distintas, tampoco la tasa de intubación bajo visión directa fue completa (aunque sí muy cercana al 100\%). Sin embargo, en el mismo se evidencia la importancia de la superioridad de la eficacia de realizar la intubación bajo visión directa y no a ciegas. Si bien en muchas circunstancias termina siendo ésta la única alternativa al no disponer de los elementos necesarios para abordar una vía aérea dificultosa, como lo demuestra Tentillier et al.[17]. Por su parte, en el trabajo publicado por Sastre et al, la tasa de intubación a ciegas a través de Fastrach es superior a I-gel[11]. 
En un estudio aleatorizado llevado a cabo por Lloyd et al.[12], en el cual formaron parte 32 anestesiólogos, la mayoría de ellos prefirió la utilización de I-gel como dispositivo guía para la intubación con BFC en vías aéreas consideradas dificultosas.

En este trabajo, dos pacientes presentaron episodios de broncoespasmo, uno de los cuales se asoció a desaturación, que revirtieron con ventilación manual y agonistas $\beta 2$ adrenérgicos inhalados sin inconvenientes; mientras que en otros dos pacientes hubo que retirar o traccionar levemente la ML para obtener un buen grado de visualización glótica (ambos acontecimientos con ML Fastrach).

La medición de la capnometría luego de retirada la ML no detectó hipercapnia en ninguno de los grupos, por lo que se infiere que la retención de $\mathrm{CO}_{2}$ es despreciable con los tiempos que se manejan para la realización de todo el procedimiento. Cabe destacar, que luego de colocada la ML y luego de realizar la IOT (todavía con la ML posicionada), los pacientes eran ventilados para evitar el colapso alveolar y la generación de atelectasias.

A modo de conclusión, se puede mencionar que los dispositivos supraglóticos pueden servir como guías para asegurar definitivamente la vía aérea, y es por eso que surge la necesidad de "combinar distintos instrumentos" con los que se cuenta para poder hacer un abordaje exitoso y seguro de la misma. Ello se ve plasmado en la tasa de éxito de intubación con BFC a través de $\mathrm{ML}$, sirviendo éstas como guías indiscutibles en personal entrenado y no entrenado en broncofibroscopía.

Aunque ambos dispositivos tuvieron la máxima tasa de éxito (tanto en su colocación como en la intubación a través de ellos), con l-gel se realizó de manera más rápida. Si bien la elección de una u otra ML no tuvieron importantes implicancias clínicas, a la hora de elegir un dispositivo se debe seleccionar aquel con el cual uno se encuentra más familiarizado y entrenado para brindar la mayor seguridad al paciente.

Agradecimientos: A los residentes e instructores de la carrera de Anestesia, Analgesia y Reanimación, del Hospital Provincial de Rosario, por la ayuda desinteresada.

A la traductora de inglés Carina Jovovic, por su ayuda en la revisión del título.

\section{Referencias}

1. American Society of Anesthesiologists Task Force on Management of the Difficult Airway. Practice guidelines for management of the difficult airway: an updated report by the American Society of Anesthesiologists Task Force on Management of the Difficult Airway. Anesthesiology. 2003 May;98(5):1269-77. https:// doi.org/10.1097/00000542200305000-00032 PMID:12717151

2. Apfelbaum JL, Hagberg CA, Caplan RA, Blitt CD, Connis RT, Nickinovich DG, et al.; American Society of Anesthesiologists Task Force on Management of the Difficult Airway. Practice guidelines for management of the difficult airway: an updated report by the American
Society of Anesthesiologists Task Force on Management of the Difficult Airway. Anesthesiology. 2013 Feb;118(2):251-70. https://doi.org/10.1097/ ALN.0b013e31827773b2 PMID:23364566

3. Crosby ET; Cooper RM; Douglas MJ; et al: The unanticipated difficult airway with recommendations for management. 1998; 45, 8:757-76.

4. Williamson JA, Webb RK, Szekely S, Gillies ER, Dreosti AV. Difficult intubation: an analysis of 2000 incidentals reports. Anaesth Intensive Care. 1993;21(5):602-7. https://doi.g/10.1177/031005 7X9302100518 PMID:8273882

5. Bertrand P, Oyarzún MA. Vía aérea difícil. Neumol. Pediatr. 2012;7(2):67-71.

6. Timmermann A. Supraglottic airways in difficult airway management: successes, failures, use and misuse. Anaesthesia. 2011 Dec;66(2 Suppl 2):45-56. https://doi.org/10.1111/ j.1365-2044.2011.06934.x PMID:22074079

7. Brain Al. The laryngeal mask-a new concept in airway management. Br J Anaesth. 1983 Aug;55(8):801-5. https://doi. org/10.1093/bja/55.8.801 PMID:6349667

8. Rodríguez Ramírez C, Bermúdez SM, Escobar IC, et al. Máscara laríngea I-Gel vs ProSeal en cirugía oncológica de mama. Rev. Cubana Anest. 2014;13:1.

9. Peterson $\mathrm{GN}$, Domino KB, Caplan RA, Posner KL, Lee LA, Cheney FW. Management of the difficult airway: a closed claims analysis. Anesthesiology. 2005 Jul;103(1):33-9. https:// doi.org/10.1097/00000542200507000-00009 PMID:15983454 
10. Añez Simón C, Boada Pié S, Solsona Dellá B. [Laryngeal mask for intubation (Fastrach)]. Rev Esp Anestesiol Reanim. 2000 Oct;47(8):352-62. PMID:11103116

11. Sastre JA, López T, Garzón JC. Intubación traqueal "a ciegas" a través de dos dispositivos supraglóticos: i-gel frente a mascarilla laríngea de intubación ILMA-Fastrach. Rev Esp Anestesiol Reanim. 2012 Feb;59(2):71-6. https://doi. org/10.1016/j.redar.2012.02.016 PMID:22480552

12. de Lloyd L, Hodzovic I, Voisey S, Wilkes AR, Latto IP; Journal of the Association of the Anaesthetists of Great Britain and Ireland. Comparison of fibrescope guided intubation via the classic laryngeal mask airway and i-gel in a manikin. Anaesthesia. 2010 Jan;65(1):3643. https://doi.org/10.1111/ j.1365-2044.2009.06155.x PMID:19891671

13. Michalek P, Donaldson W, Graham C, Hinds JD. A comparison of the I-gel supraglottic airway as a conduit for tracheal intubation with the intubating laryngeal mask airway: a manikin study. Resuscita- tion. 2010 Jan;81(1):74-7.

https://doi.org/10.1016/j.

resuscitation.2009.10.009

PMID:19926388

14. Moore A, Gregoire-Bertrand F, Massicotte N, Gauthier A, Lallo A, Ruel $M$, et al. I-gel versus LMA-Fastrach Supraglottic Airway for Flexible Bronchoscope-Guided Tracheal Intubation Using a Parker (GlideRite) Endotracheal Tube: A Randomized Controlled Trial. Anesth Analg. 2015 Aug;121(2):430-6. https://doi.org/10.1213/ ANE.0000000000000807 PMID:26076387

15. Díez LD, Barrado Muñoz L, Blanco $P$, et al. La capnografía en los servicios de emergencia médica. Semergen. 2009;35(3):138-43. https://doi.org/10.1016/\$11383593(09)70721-X.

16. Tusman $G$, Suárez-Sipmann $F$, Bohm SH, Borges JB, Hedenstierna G. Capnography reflects ventilation/perfusion distribution in a model of acute lung injury. Acta Anaesthesiol Scand. 2011 May;55(5):597-606. https://doi.org/10.1111/ j.1399-6576.2011.02404.x PMID:21342153

17. Tentillier $E$, Heydenreich $C$, Cros AM, Schmitt V, Dindart JM, Thicoïpé $M$. Use of the intu- bating laryngeal mask airway

in emergency pre-hospital

difficult intubation. Resuscitation. $2008 \mathrm{Apr} ; 77(1): 30-4$. https://doi.org/10.1016/j. resuscitation.2007.06.035 PMID:18022753

18. Theiler LG, Kleine-Brueggeney $M$, Kaiser D, Urwyler N, Luyet $C$, Vogt A, et al. Crossover comparison of the laryngeal mask supreme and the i-gel in simulated difficult airway scenario in anesthetized patients. Anesthesiology. 2009 Jul;111(1):55-62. https://doi.org/10.1097/ ALN.0b013e3181a4c6b9 PMID:19512881

19. American Collegue of Surgeons. PHTLS: Soporte vital básico y avanzado en el trauma prehospitalaria. 7th ed. Barcelona: Elsevier España; 2012.

20. Ostermayer DG, Gausche-Hill M Los dispositivos supraglóticos de la vía aérea: historia y situación actual de los dispositivos prehospitalarios de la vía aérea. PrehospEmergCare. Edición en español. 2014, 2:1-12

21. Brimacombe J; Berry A: A proposed fiber-optic scoring system to standardize the assessment of laryngeal mask position. AnestAnalg. 1993, 76, 2: 457. 